\section{Transformation of a myelodys- plastic syndrome to acute myeloid leukemia and concurrent necrotizing sweet syndrome}

\author{
Olga Vera-Lastra, ${ }^{1,2}$ Arturo Olvera- \\ Acevedo, ${ }^{1,2}$ Nancy Pulido-Díaz, ${ }^{3}$ \\ Marissa de Jesús Quintal-Ramírez, ${ }^{4}$ \\ Irvin Ordoñez-González, ${ }^{5}$ \\ Erik Cimé-Aké, ${ }^{1,2}$ María Pilar Cruz- \\ Domínguez, ${ }^{6,2}$ Gabriela Medina ${ }^{7,2}$ \\ ${ }^{1}$ Internal Medicine Department, \\ Hospital de Especialidades Centro \\ Médico Nacional La Raza, IMSS, \\ Mexico City; ${ }^{2}$ Universidad Nacional \\ Autónoma de México, Mexico City; \\ ${ }^{3}$ Dermatology Department, Hospital de \\ Especialidades Centro Médico Nacional \\ La Raza, Instituto Mexicano del Seguro \\ Social, Mexico City; ${ }^{4}$ Pathology \\ Department, Hospital de Especialidades \\ Centro Médico Nacional La Raza, \\ Instituto Mexicano del Seguro Social, \\ Mexico City; ${ }^{5}$ Universidad Veracruzana, \\ Veracruz; ${ }^{6}$ Translational Research Unit, \\ Hospital de Especialidades Centro \\ Médico Nacional La Raza, Instituto \\ Mexicano del Seguro Social, Mexico \\ City; ${ }^{7}$ Research Division, Hospital de \\ Especialidades Centro Médico Nacional \\ La Raza, Instituto Mexicano del Seguro \\ Social, Mexico City, Mexico
}

\begin{abstract}
The Sweet's syndrome, is an inflammatory skin disorder characterized by extensive infiltration of neutrophils in the dermis with extension to the subcutis, known as acute febrile neutrophilic dermatosis. It may occur as a paraneoplastic syndrome. To our knowledge, there are currently few reports about transformation of a myelodysplastic syndrome to acute myeloid leukemia and concurrent necrotizing Sweet syndrome in the literature. Herein we describe an unusual case in a young patient with these characteristics that evolved to a fatal outcome.
\end{abstract}

\section{Introduction}

The Sweet's syndrome (SS), is an inflammatory skin disorder characterized by extensive infiltration of neutrophils in the dermis with extension to the subcutis, known as Acute febrile neutrophilic dermatosis.

The clinical spectrum of manifestations is wide, some of the most representative characteristics are tender skin lesions, erythematous plaques, papules or nodules located in extremities, neck and head, usually accompanied by fever and neutrocytosis. ${ }^{2}$ Some of the clinical conditions related to this syndrome include infections, autoimmune diseases, inflammatory bowel diseases, vaccines, pregnancy, certain medications, neoplasms and idiopathic. ${ }^{3}$

Paraneoplastic syndromes are clinical clues that neoplasms cause in places outside their primary location and that are directly associated with them or with their metastases. They may occur accompanying an established cancer or as the first sign of malignancy or its recurrence. ${ }^{4}$

The malignancy-associated Sweet's syndrome (MASS) can occur as a paraneoplastic syndrome in patients with solid tumors such as carcinomas of the genitourinary tract, breast, and gastrointestinal tract or related to hematologic condition including myeloproliferative, lymphoproliferative, and myelodysplastic disorders. Among the hematologic malignancies most commonly associated with Sweet's Syndrome is Acute Myeloid Leukemia (AML). ${ }^{5}$

Herein, we report a case of Myelodysplastic Syndrome (MS) that evolved to AML with concurrent SS as a skin paraneoplastic condition.

\section{Case Report}

A 23-years-old female patient, with a history of marijuana addiction for one year, tobacco and alcohol consumption since the age of 17 , three abortions before 10 weeks of gestation, previously diagnosed in May 2012, with Human Papillomavirus (HPV) infection by cervical cytology and aplastic anemia by bone marrow aspiration (BMA). She received treatment with Antithymocyte Globulin for 2 cycles (May 2012 and April 2014) and posteriorly Cyclosporin A, with subsequent liver toxicity, changing to Mycophenolate Mofetil and Danazol, with a partial response, requiring multiple hospitalizations and transfusion support, with more than 40 units of globular packages, complicating with transfusional hemosiderosis, treated with Deferasirox. In May 2015, she presented cellulitis of the right thoracic limb, with spontaneous resolution and a secondary atrophic scar. In November 2015, she began with asthenia, adynamia and fever up to $41^{\circ} \mathrm{C}$, predominantly in the evening and a nodular lesion of $3 \mathrm{~cm}$ diameter in the upper inner quadrant (UIQ) of the right breast, which increased in size progressively, with very painful violaceous
Correspondence: Gabriela Medina García, Hospital de Especialidades Centro Médico Nacional "La Raza", Seris y Zaachila S/N, Colonia La Raza, Alcaldía Azcapotzalco, Ciudad de México, 02990, Mexico.

Tel.: +52.5557245900

E-mail: dragabymedina@yahoo.com.mx

Key words: Necrotizing Sweet syndrome, Myelodysplastic syndrome, Acute myeloid leukemia.

Contributions: OVL: formulating the research problem and medical clinical evaluation. AOA: medical clinical evaluation. NPD: dermatologist. MJQR: pathologist. IOG: writing the first original draft, review and editing. ECA: data collection and review of literature. MPCD: critical review of the manuscript. GM: writing, critical review of the manuscript and corresponding author.

Conflict of interest: The authors declare no conflict of interests.

Ethics approval: We obtained the approval of local Ethics comitee.Personal data from the patient are not revealed in the manuscript

Consent to publication: Due to the fact that the patient died,we obtained consent to publication from her relatives.

Please cite this article as: Vera-Lastra $O$. Olvera-Acevedo A., Pulido-Díaz N, et al. Transformation of a myelodysplastic syndrome to acute myeloid leukemia and concurrent necrotizing sweet syndrome. Dermatol Rep 2021;13:9017.

Received for publication: 13 November 2020 . Revision received: 11 February 2021.

Accepted for publication: 15 February 2021.

This work is licensed under a Creative Commons Attribution-NonCommercial 4.0 International License (CC BY-NC 4.0).

${ }^{\circ}$ Copyright: the Author(s), 2021

Licensee PAGEPress, Italy

Dermatology Reports 2021; 13:9017

doi:10.4081/dr.2021.9017

skin lesions, evolving to confluent blisters and later ulceration with non-purulent secretion and necrosis (Figure 1A). She was treated with Dicloxacillin, Piperacillin / Tazobactam and Carbapenemic. In the absence of improvement, stage IIIB breast cancer was suspected, so she was referred in December 2015 to a tertiary level hospital. Upon admission, it was documented the lesion in the right breast, with bleeding nipple, as well as confluent blisters and edema on the outer side of the left thigh (Figure $1 \mathrm{~B}, \mathrm{C})$. The patient had a non-reactive viral 
panel for HIV, HCV, HBV. A breast skin biopsy revealed a neutrophilic inflammatory infiltrate with no evidence of vasculitis (Figure 2). Treatment with systemic steroid was started, with excellent response and remission of lesions. Imaging tests were performed, as chest x-ray and Thoracoabdominal computed tomography, finding hepatosplenomegaly, the final diagnosis was necrotizing Sweet's syndrome. A month later, a new BMA was performed, finding dysplasia of more than $50 \%$ of the cells of the erythroid and granulocytic series, as well as absent megakaryocytes, diagnosing MS. The patient persisted with transfusion support with globular packages and treatment with Mycophenolic Acid. On November 2016, the patient was hospitalized for a 2-week course consistent with fever, anemia and purpuric syndrome, low cardiac output data, syncope, and acute kidney injury. BMA congruent for AML with absent megakaryocytes, $7 \%$ adult neutrophils, $2 \%$ young neutrophils, $1 \%$ lymphocytes, $77 \%$ normoblasts, $13 \%$ myeloid blasts and 58\% myeloid blasts. Immunophenotyping: $32.6 \%$ HLA-DR, 29.2\% CD34, 36.5\% Glycophorin, 2.08\% Glycophorin A/34, 28.6\% CD117, 53.8\% CD13, 51\% CD33, 12,7\% CD64, 1.9\% CD7 and 31.6\% CD22. Management began with anti-tumor lysis measures, hemoderivatives, and leukapheresis. Five days after admission, the patient presented respiratory failure, requiring mechanical ventilatory support, progressing to multiple organ failure culminating in death, with final diagnoses of AML subtype M2, aplastic anemia and Sweet's Syndrome.

\section{Discussion}

We described a case in a young patient with aplastic anemia that evolved to myelodysplastic syndrome and further to acute myeloid leukemia with necrotizing Sweet syndrome as a paraneoplastic expression of the latter. This hematologic disorder is the most common associated malignancy, being important to carry out the differential diagnosis since breast cancer was initially suspected. $^{5}$

Hematologic disorders represent more than $15-20 \%$ of MASS, being AML and MS the most common. ${ }^{6}$

Few cases coincident with MS, AML and SS have been reported in the literature as the case of a 15-year-old girl who presented these three entities with FLT3 and NPM1 type A mutations. Risk factors for MASS in AML encompass deletion of chromosome 5 or $5 \mathrm{q}$, presence of FLT3 muta- tions, and AML with myelodysplasia-related features. Unfortunately, our patient did not have a karyotype due to a rapid adverse clinical course. ${ }^{7}$

Pourmoussa and Kwan reported another case of an extremely rapid transformation from MS with concomitant SS to AML, in an elderly patient. ${ }^{8}$ Our patient also followed that sequence with a fatal ending. Myelodysplastic Syndrome can evolve into AML, which often leads to a poor prognosis.

The outlook of SS is little known, with an incidence of 2.7 at 3 cases/100,000 in the general population. ${ }^{9}$ The diagnosis of SS associated with MS with transformation to AML has a low incidence and it is scarcely described in the literature, but of importance in the diagnostic suspicion.

In Mexico, a recent multicenter study identified that AML presents at a younger age in comparison with developed countries, with a median age of onset of 47 years, however this patient presented AML at an earlier age. ${ }^{10}$

SS related to hematological neoplasia may present prior to or concomitant to the primary diagnosis, that means a paraneoplastic event, in the patient occurred concomitantly with acute myeloid leukemia, similar to that reported by Mo et al. ${ }^{11}$

Necrotizing SS or acute necrotizing neutrophilic dermatosis is an infrequent and severe variant, distinguished by aggressive skin lesions that can easily simulate and be mistaken for necrotizing soft tissue infections such as necrotizing fasciitis or pyoderma gangrenosum. It is characterized by hyperpyrexia, neutrophilia and painful skin lesions that can be single or multiple, be vesicular, pustular, bullous or ulcerative, and mainly necrosis. ${ }^{12}$

The term Necrotizing SS proposed in 2012 and to date only 4 cases have been reported worldwide, in which patients had concealed hematological diseases and histopathological findings with necrosis of the fascia and fat, simulating necrotizing

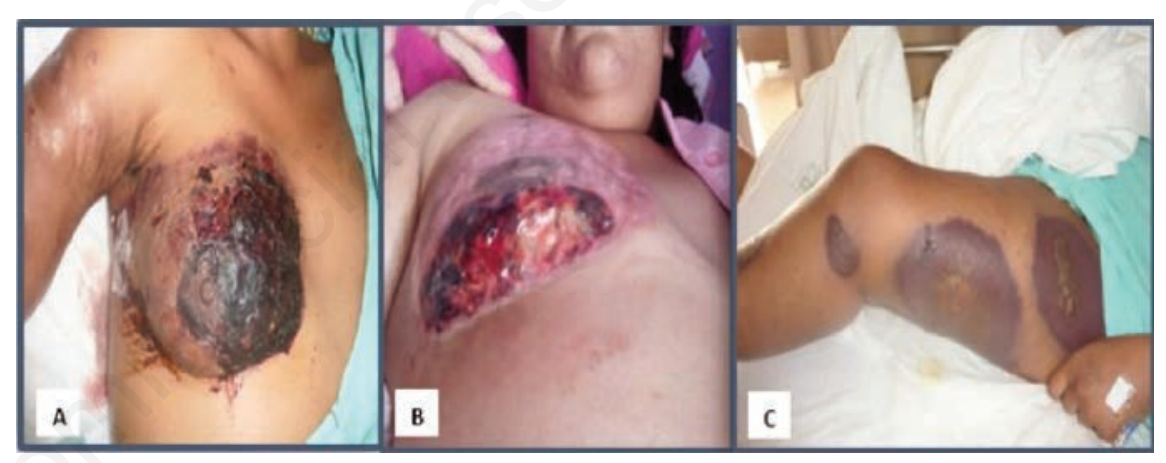

Figure 1. A) Cutaneous necrosis of the right breast. B) Erythematous violaceous plaque with undefined edges, ulcerated with perilesional edema and purpuric raised edges, central blisters, erosion and bleeding nipple; 1C. Lesions on the outer side of the left thigh characterized by confluent blisters and peripheral edema.

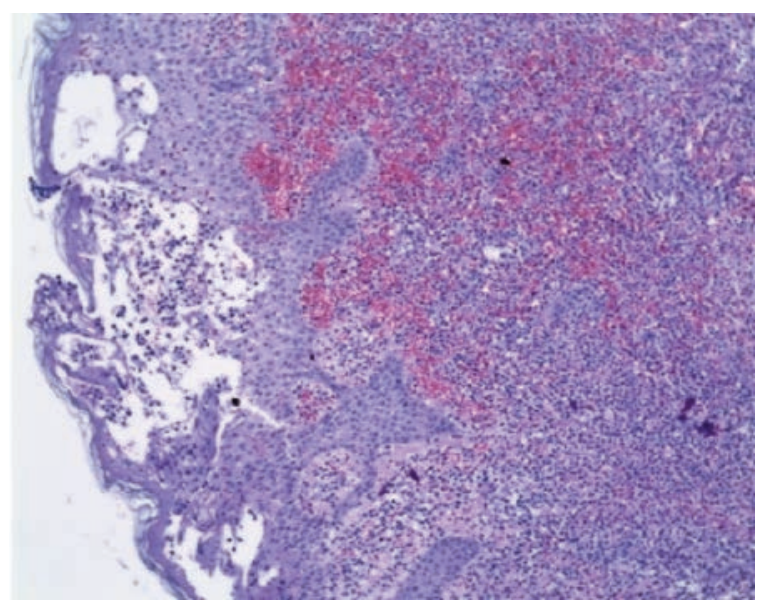

Figure 2. Superficial and deep neutrophilic dermatosis, with spongiform pustules, secondary neutrophilic vascular reaction in small vessels, and added purpuric phenomenon. 
fasciitis. Previous cases were males with a median age of 57.5 years of onset, all of them with underlying hematological diseases, however the present case was in a very young woman with an aggressive clinical course. ${ }^{13}$

This entity may be confused with other pathologies, among the most common differential diagnoses that mimic SS are bacterial, mycobacterial, fungal, and parasitic infections. To avoid this, a biopsy should be considered to detect characteristic features consistent with SS. ${ }^{5}$ This disease has a low incidence and is little described in the literature. Communications of fatal outcomes of Sweet's syndrome are uncommon, as it is depicted as an idiopathic chronic systemic inflammatory response syndrome or as in this case, related to malignancy with a fatal outcome in a young patient. ${ }^{1,14}$

\section{Conclusions}

Sweet syndrome is a rare entity that may appear as a sign of malignancy, as in the present case, in a young patient with fatal outcome. It is necessary to have a high index of suspicion to recognize it and identify the underlying disorder.

\section{References}

1. Villarreal CD, Ocampo J, Villarreal A. Sweet Syndrome: A review and update. Actas Dermosifiliogr 2016;107:369-78.

2. Zheng S, Li S, Tang S, et al. Insights into the characteristics of Sweet Syndrome in patients with and without hematologic malignancy. Front Med 2020;7:1-5.

3. Dan H, Yanmei L, Yue X, Xian J. Sweet syndrome associated with malignancies: A retrospective analysis of 25 patients from West China hospital. Dermatol Ther 2020;1:1-14.

4. Gomes D, Campos G, Macchione J, Costa G. Paraneoplastic Sweet's syndrome. Ann Bras Dermatol 2018;93:576-8.

5. Clark AK, Sarcon AK, Fung MA, et al. Malignancy-associated Sweet syndrome: acute febrile neutrophilic dermatosis associated with recurrence of metastatic cervical cancer. Dermatol Online J 2017;23:1-5.

6. Cohen PR, Kurzrock R. Sweet's syndrome and malignancy. Am J Med 1987;82:1220-6.

7. Chen SH, Kuo YT, Liu YL, et al. Acute myeloid leukemia presenting with Sweet syndrome: A case report and review of the literature. Pediatr
Neonatol 2016;58:283-4.

8. Pourmoussa A, Kwan K. An unlikely rapid transformation of myelodysplastic syndrome to acute leukemia: A case report. Perm J 2017;21:16-91.

9. Djien V, Wallach D. Sweet's syndrome: practical conduct. Ann Dermatol Venereol 1999;126:343-7.

10. Demichelis R, Zapata N, Leyto F, et al. Acute myeloid leukemia in Mexico: the specific challenges of a developing country. Results from a multicenter national registry. Clin Lymphoma Myeloma Leuk 2019;20:e295-w303.

11. Mo W, Wang X, Wang Y, et al. Clonal neutrophil infiltrates in concurrent Sweet's syndrome and acute myeloid leukemia: A case report and literature review. Cancer Genet 2018;226:11-6.

12. Lipof JS, Beck LA, Reddy SC, et al. Necrotizing Sweet Syndrome of the Upper Extremity After Elective Hand Surgery. J Hand Surg Am 2018;43:e1-6.

13. Minamiyama R, Asai J, Kaneko Y, et al. Necrotizing Sweet syndrome in a patient with myelodysplastic syndrome. Clin Exp Dermatol 2017;42:436-8.

14. Rochet NM, Chavan RN, Cappel MA, et al. Sweet syndrome: clinical presentation, associations, and response to treatment in 77 patients. J Am Acad Dermatol 2013;69:557-64. 